\title{
Detection of Firewall Fingerprinting and Vulnerability Prevention by Denial of Attacks on Web Application
}

\author{
Dilli Babu $\mathbf{M}^{1}$, Balamani $M^{2}$, Mukesh $\mathbf{G}^{3}$, Ajay Krishna $S^{4}$, Kasi Rajan $\mathrm{B}^{5}$ \\ Assistant Professor, Department of Information Technology, Panimalar Engineering College, Chennai, India ${ }^{1}$ \\ Student, Department of Information Technology, Panimalar Engineering College, Chennai, India ${ }^{2,3,4,5}$
}

\begin{abstract}
Firewalls are most important and critical devices which provides securities against all vulnerabilities. Firewall handles all the traffic in and out of the network. We think firewall is secure but it's not many vulnerabilities compromise the firewalls. Hackers / intruders exploit the firewall (host based) using malicious scripts and access the server / applications. In this project, we analyse firewall finger printing and denial of firewalling. We also analyze attacks namely path traversal, DOM XSS, file inclusion, CSRF which are bye passed beyond and we handle those attacks. Our proposed system provides efficient fingerprinting methods to prevent the attacks. Also, the proposed system provides security against application as well. If the firewall is been compromised, intruder can access the files in the application or server because application is made secure against most common web vulnerabilities. This security on application is achieved to prevent the above attacks. Thus, our proposed system of firewall fingerprinting methods can achieve quite high accuracy against all web vulnerability. Thus, all web applications can be made secure against web attacks.
\end{abstract}

Keywords: Firewall, Vulnerabilities, Fingerprinting, Secure

\section{INTRODUCTION}

\section{A. $\quad$ Purpose of system}

The main aim of this project is to provide an security on application is achieved to prevent the above attacks and Vulnerability.

\section{B. $\quad$ Project scope}

The scope of the project is to provide an environment to secure against all attacks and vulnerabilities.web application topic is crucial for our success, sometimes even for the survival. the critical security in companies are cross side scripting (XSS) and SQL Injection(SQLi) or directory traversal. To attack these vulnerabilities the attacker send the rogue request to the vulnerable web applications. If the application confuses the payloads of the rogue requests and commands. the attack succeeded and attacker can edit, delete and change the sensitive information in the application. To prevent these attacks, we have implanted this project to secure the database from the illegal hackers and safeguard the system.

\section{C. $\quad$ Firewall approach}

In this paper ,for the first we have proposed some set of techniques that can collect some information about each firewall packet processing time of probe packets are used to implement the firewall. For one computer and measuring the firewall processing time of the probe packets. Because firewalls are very expensive and due to privacy and legal reasons we are obligated to keep brand and model of firewall in more confidential.

\section{LITERATURE SURVEY}

\section{A. $\quad$ Study of SQL Injection Attacks and Countermeasures}

In this paper we proposes, SQL injection is an attack technique that exploits a security vulnerability occurring in the database layer of an application and a service. This is most often found within web pages with dynamic content. This paper provides taxonomy on SQL injection prevention and detection approaches. Furthermore, for each type of vulnerability, we provide descriptions of how attacks of that type could take advantage of that vulnerability and perform attack. We also present and analysis some of existing detection and prevention techniques against SQL injection attacks. Finally, we compare different type of approaches and techniques and provide a list of their deployment requirements. 


\section{International Journal of Advanced Research in Computer and Communication Engineering}

Vol. 8, Issue 2, February 2019

B. A Classification of SQL Injection Attacks and Countermeasures

In this paper, SQL injection attacks pose a serious security threat to Web applications: they allow attackers to obtain unrestricted access to the databases underlying the applications and to the potentially sensitive information these databases contain. Although researchers and practitioners have proposed various methods to address the SQL injection problem, current approaches either fail to address the full scope of the problem or have limitations that prevent their use and adoption. Many researchers and practitioners are familiar with only a subset of the wide range of techniques available to attackers who are trying to take advantage of SQL injection vulnerabilities. As a consequence, many solutions proposed in the literature address only some of the issues related to SQL injection. To address this problem, we present an extensive review of the different types of SQL injection attacks known to date. For each type of attack, we provide descriptions and examples of how attacks of that type could be performed. We also present and analyse existing detection and prevention techniques against SQL injection attacks. For each technique, we discuss its strengths and weaknesses in addressing the entire range of SQL injection attacks.

\section{An Approach to Detect and Prevent SQL Injection Attacks in Database Using Web Service}

In this paper we proposes, SQL injection is an attack methodology that targets the data residing in a database through the firewall that shields it. The attack takes advantage of poor input validation in code and website administration. SQL Injection Attacks occur when an attacker is able to insert a series of SQL statements in to a 'query' by manipulating user input data in to a web-based application, attacker can take advantages of web application programming security flaws and pass unexpected malicious SQL statements through a web application for execution by the backend database. This paper proposes a novel specification-based methodology for the prevention of SQL injection Attacks. The two most important advantages of the new approach against existing analogous mechanisms are that, first, it prevents all forms of SQL injection attacks; second, Current technique does not allow the user to access database directly in database server. The innovative technique "Web Service Oriented XPATH Authentication Technique" is to detect and prevent SQL Injection Attacks in database the deployment of this technique is by generating functions of two filtration models that are Active Guard and Service Detector of application scripts additionally allowing seamless integration with currently-deployed systems.

\section{D. $\quad$ A Survey of SQL Injection Attack Detection and Prevention}

In this paper, Structured Query Language Injection Attack (SQLIA) is the most exposed to attack on the Internet. From this attack, the attacker can take control of the database therefore be able to interpolate the data from the database server for the website. Hence, the big challenge became to secure such website against attack via the Internet. We have presented different types of attack methods and prevention techniques of SQLIA which were used to aid the design and implementation of our model. In the paper, work is separated into two parts. The first aims to put SQLIA into perspective by outlining some of the materials and researches that have already been completed. The section suggesting methods of mitigating SQLIA aims to clarify some misconceptions about SQLIA prevention and provides some useful tips to software developers and database administrators. The second details the creation of a filtering proxy server used to prevent a SQL injection attack and analyses the performance impact of the filtering process on web application.

\section{SYSTEM ANALYSIS}

\section{A. $\quad$ Existing System}

We think firewall is secure but it's not many vulnerabilities compromise the firewalls. Hackers / intruders exploit the firewall using malicious scripts and access the server / applications. Reflected Cross-Site Scripting Attacker goal: their code into browser XSS forces a website visitor to execute malicious code in his/her browser. There is no deployed technology that has successfully defended against DDOS attacks. Most of the approaches focus, perhaps understandably, on protection of customer sites against incoming attacks. This turns out to be very difficult to do with today's Internet architecture and protocols

\section{B. $\quad$ Proposed System}

In this work, The proposed system focuses on how to detect and prevent SQL injection attacks on web applications using encryption and tokenization technique. The tokenization process is applied on the input query by detecting spaces, single quotes and double dashes etc. This process converts the input query into fruitful tokens and that are stored in a dynamic table at the client side. This process converts the input query into fruitful tokens and that are stored in a dynamic table at the client side. The table name, field name and data are encrypted using cryptography algorithm. The encrypted original input query and the tokenized table are sending to the server side. At the server side, input query is decrypted and in turn converts into various token which are stored in to another dynamic table. Both dynamic tables are compared and if both are equal, it seems that there is no injection attacked in the given query, hence the query is proceeding further to main database for retrieving result. 


\section{SYSTEM DESIGN}

A. $\quad$ System Architecture

System architecture is the conceptual model that defines the structure, behaviour, and more views of a system. An architecture description is a formal description and representation of a system, organized in a way that supports reasoning about the structures and behaviours of the system. Figure 1 shows the architecture.

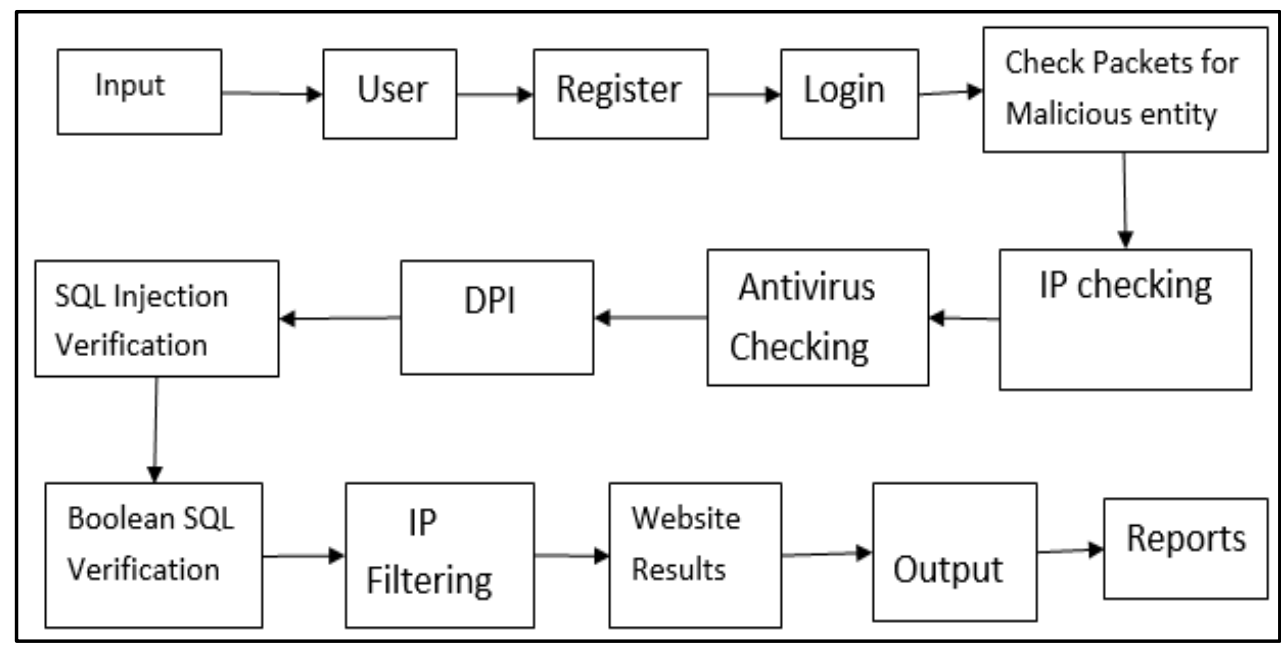

Fig. 1 Architecture

\section{MODULES}

The proposed system consists of four main modules. They are:

- $\quad$ Standard sql injection

- Broken authentication

- $\quad$ http banner disclosure

- Deep packet inspection

A. Standard sql injection

SQL injection is a code injection technique, used to attack data-driven applications, in which malicious SQL statements are inserted into an entry field for execution (e.g. to dump the database contents to the attacker).SQL injection must exploit a security vulnerability in an application's software, for example, when user input is either incorrectly filtered for string literal escape characters embedded in SQL statements or user input is not strongly typed and unexpectedly executed. SQL injection is mostly known as an attack vector for websites but can be used to attack any type of SQL database.

\section{B. $\quad$ Broken authentication}

Authentication and session management includes all aspects of handling user authentication and managing active sessions. Authentication is a critical aspect of this process, but even solid authentication mechanisms can be undermined by flawed credential management functions, including password change, forgot my password; remember my password, account update, and other related functions. Because "walk by" attacks are likely for many web applications, all account management functions should require re-authentication even if the user has a valid session id.

\section{C. $\quad$ Http banner disclosure}

Banner Grabbing is an enumeration technique used to glean information about computer systems on a network and the services running its open ports. Administrators can use this to take inventory of the systems and services on their network.. An intruder however can use banner grabbing in order to find network hosts that are running versions of applications and operating systems with known exploits.

\section{Deep packet inspection}

In this module automatic Intrusion Detection System (IDS), encryption, deep packet inspection (DPI) and report the results to the controller. The main goal of this module is to allow network operators to describe security policies for specific flows. The policies include a description of the flow, a list of security services that apply to the flow and how to react in case malicious content is found. The reaction can be to alert only, or to quarantine traffic or even block all packets from a specific source. 


\section{IJARCCE}

Vol. 8, Issue 2, February 2019

\section{CONCLUSION}

We present methods for finding the firewall characteristics that are introduced by firewall implementations. Such characteristics can be exploited by attackers to identify black box firewalls with high accuracy and launch effective attacks on firewalls. We further study the impact of different attacks on different firewalls and show that different firewalls are vulnerable to different attacks. To evaluate the effectiveness of defence mechanisms and measure their impact on firewall performance, we need to conduct extensive experiments, for which we will need to expand our test bed.

\section{REFERENCES}

[1]. Avireddy. S, Perumal.V, Gowraj.N,Kannan R.S, Thinakaran.P, Ganapthi .S, Gunasekaran J.R, Prabhu.S, Random4: An Application Specific Randomized Encryption Algorithm to prevent SQL injection, ieee transactions on communications, vol. 60, no. 5, may 2012.

[2]. Debabrata Kar, SuvasiniPanigrahi, Prevention of SQL Injection Attack Using Query Transformation and Hashing, IEEE International Advance Computing Conference (IACC), 2013.

[3]. Gaurav Shrivastava, Kshitij Pathak, SQL Injection Prevention using Tokenization: Technique and Prevention Mechanism, IJARCSSE, Volume 3, Issue 6, June 2013.

[4]. Ke Wei, M. Muthuprasanna, Suraj Kothari, "Preventing SQL Injection attacks in Stored Procedures".Proceedings of the 2006 Australian Software engineering Conference (ASWEC ${ }^{\text {ee }} 06$ ).

[5]. "Cisco Firewall Services Module DoS vulnerability", http://www.netsecurity.org/secworld.php?id=10673, 2011.

[6]. Zhiyun Qian and Z. Morley Mao, "Off-path tcp sequence number inference attack - how firewall middleboxes reduce security", in Proceedings of the IEEE Symposium on Security and Privacy, Oakland, California, May 2012, pp. 347 - 361.

[7]. Fyodor, "Nmap: Free network security scanner", http://nmap.org.

[8]. Fedor V. Yarochkin, Ofir Arkin, MederKydyraliev, Shih-Yao Dai, Yennun Huang, and Sy-Yen Kuo, "Xprobe2++: Low volume remote network information gathering tool", in Proceedings of the DSN, 2009 